\title{
A randomized $3 \times 3$ crossover study to evaluate the effect of Hass avocado intake on post-ingestive satiety, glucose and insulin levels, and subsequent energy intake in overweight adults
}

Michelle Wien ${ }^{1 *}$, Ella Haddad ${ }^{1}$, Keiji Oda ${ }^{2}$ and Joan Sabaté ${ }^{1,2}$

\begin{abstract}
Background: The behavioral outcome of food ingestion is a complex process that involves psychological and biological factors. Avocados are nutrient dense with properties that may favorably impact energy balance. This study sought to evaluate if incorporating approximately one half of a Hass avocado by addition or inclusion into a lunch meal will influence post-ingestive satiety, glucose and insulin response, and subsequent energy intake among overweight adults.

Methods: This was a randomized $3 \times 3$ single-blind crossover design study with 26 healthy overweight adults (mean \pm SD age $40.8 \pm 11.0$ years and BMI $28.1 \pm 2.4 \mathrm{~kg} / \mathrm{m}^{2}$ ). Participants consumed a standardized breakfast followed by 1 of 3 lunch test meals [Control (C), avocado-free; Avocado Inclusive (Al); and, Avocado Added (AA)]. Participants rated five appetite sensations using a visual analog scale (VAS) before lunch and at specific intervals over 5 hours following the start of the test meal. Blood glucose and insulin were measured before lunch and at specific intervals over 3 hours following the start of the test meal. Mixed models were used to compare differences among the 3 test meals, and the area under the curve $\left(A \cup C_{0-x h}\right.$ ) was computed for the VAS and biological measures.
\end{abstract}

Results: There were significant differences in the $A \cup C_{(0-5 h)}$ for the self-reported feelings of satisfaction ( $\left.P=0.04\right)$ and desire to eat $(\mathrm{P}=0.05)$ in the mixed model analysis. Compared to the $C$ test meal, the AA test meal increased satisfaction by $23 \%(P=0.05)$ and decreased the desire to eat by $28 \%(P=0.04)$ for the $A \cup C_{(0-5 h)}$. For the $A \cup C_{(0-3 h)}$, the $A A$ test meal increased satisfaction by $26 \%(P=0.02)$ and decreased the desire to eat by $40 \%(P=0.01)$ as compared to the $\mathrm{C}$ test meal. Compared to the $\mathrm{Al}$ meal, the $A \cup \mathrm{C}_{(0-3 \mathrm{~h})}$ for blood insulin was higher in the $\mathrm{C}$ and $\mathrm{AA}$ meals ( $P=0.04$ and $P=0.05$, respectively).

Conclusions: The addition of approximately one half of a Hass avocado at a lunch meal can influence post-ingestive satiety over a subsequent 3 and 5 hour period in overweight adults. A caveat to these findings is that the avocado contained an additional $112 \mathrm{kcal}$, which may have accounted for the observed increase in satisfaction and decreased desire to eat. Future trials are warranted to evaluate the effects of avocado intake on weight management in adults of varying BMls and among insulin resistant individuals.

Keywords: Avocado, Satiety, Overweight, Glucose, Insulin, Visual analog scale, Dietary compensation

\footnotetext{
* Correspondence: mwien@llu.edu

${ }^{1}$ Department of Nutrition, School of Public Health, Loma Linda University,

24951 N. Circle Dr., Nichol Hall 1102, Loma Linda, CA 92350, USA

Full list of author information is available at the end of the article
} 


\section{Background}

The behavioral outcome of food ingestion is a complex process that involves psychological and biological factors that culminates in an individual's overall 24-hour energy intake [1]. One of the components of the appetite system is satiety, which reflects a process that leads to increased fullness after a meal, a decline in hunger, and inhibition of further eating in the postprandial period.

In addition to sensory quality, the macronutrient composition, physical structure and energy density of a whole food may contribute to the modulation of satiety. More recent research has shown that the volume of a meal also influences satiety [2,3]. Additionally, the quality of the fat composition, i.e. degree of saturation of fatty acids in a food, may influence rates of oxidation and thermogenesis in animals and humans [4,5]. Furthermore, a single food may favorably impact energy balance according to its ability to offset spontaneous energy reduction at the next meal(s), which is known as the dietary compensation score [6].

The fresh pulp weight of Hass avocados is $72 \%$ water and contains only $1.7 \mathrm{kcal} / \mathrm{g}$, therefore they are classified as a medium energy dense food (defined as a range between 1.5-4.0 kcal/g) [7]. Hence, when added to a meal they will increase the volume similar to other fruits and vegetables, which are food categories that have been previously shown to have a beneficial effect on weight control [8]. Further, the typical serving size is approximately one half of a medium size avocado (70 g) [9], which translates to being an excellent source of dietary fiber $(5 \mathrm{~g})$. Fiber is another food component strongly linked to enhancement of satiety [10] and modulation of the glucose and insulin responses to meals [11].

The connection between potential biological markers of appetite regulation continues to be an active area of research in normal weight and overweight populations. A 2007 meta-analysis by Flint et al. [12] has shown that the biological signaling of satiety by insulin in the overweight population is blunted, which could lead to the deleterious consequence of overeating at subsequent ad libitum meals and snacks. In light of the increased prevalence in overweight in humans and cross-sectional evidence showing an increase in snacking and total energy intake in the United States over the past three decades [13], the addition of approximately one half of an avocado at a specific meal(s) may be a simple dietary intervention to consider for individuals that consume excessive energy during specific snack and/or meal times. The aims of the present study are to evaluate if incorporating $\sim 1 / 2$ of a fresh Hass avocado by addition or inclusion into a lunch meal will influence postingestive satiety, the glycemic and insulin response, and subsequent energy intake in overweight adults.

\section{Methods}

We conducted a randomized 3x3 single-blind crossover design study (three 1-day study periods scheduled 1 week apart) at Loma Linda University, Loma Linda, California. Using a within subject repeated measures design, we evaluated the effect of avocado intake on the short-term regulation of food by employing the use of one of three lunch test meals within a single day on three different days. Each participant received all treatments on the same day of the week and had a 1 week washout period between treatments.

\section{Eligibility criteria}

Healthy overweight and moderately obese adults were recruited through the use of posters, flyers, and newspaper advertisements on the Loma Linda University campus and in the surrounding communities. A study web page was developed with a complete description of the study and online application form. Applications were also taken by phone.

Eligibility criteria were: age 25-65 years, body mass index (BMI, $\mathrm{kg} / \mathrm{m}^{2}$ ) $\geq 25$ and $\leq 35$, weight stable for at least 6 months, normoglycemic, normotensive, sedentary or low level of habitual activity (less than 10 hours of exercise per week), non-smoker, not dependent on caffeine, and not taking any medications known to influence postprandial glucose and insulin levels. The recruitment process yielded 80 applicants and 56 individuals met the eligibility criteria. Forty-seven individuals attended information meetings and 30 were selected, plus 2 alternates. Four of the selected applicants declined participation due to unforeseen scheduling conflicts. Both alternates were included to achieve the targeted accrual of 28 participants and goal of 25 completers for adequate power (see Statistical Methods). One participant withdrew on the first day and one participant was asked to leave the second week due to non-compliance with the study protocol. The study was approved by the Loma Linda University Institutional Review Board and informed written consent was obtained from all participants.

\section{Anthropometric measurements}

Height was measured to the nearest centimeter using a stadiometer on the first study day. Weight was measured using an internally calibrated segmental body composition scale/analyzer (model TBF-300A, Tanita ${ }^{\circledR}$, Arlington Heights, IL) and recorded to the nearest 0.01 pound. BMI was calculated as weight $(\mathrm{kg}) /$ height $\left(\mathrm{m}^{2}\right)$. The daily energy needs for each participant were estimated using the Harris-Benedict equation after adjustment for overweight status, which was subsequently multiplied by an activity factor of 1.3 for sedentary lifestyle. Participants were then assigned to receive either a 1600, 2000 or 2400 kcal daily meal plan. 


\section{Study meals}

Under the direct supervision of trained study personnel in the Loma Linda University Department of Nutrition Metabolic Kitchen, participants consumed the same standardized breakfast meal containing $25 \%$ of their estimated daily energy needs on each of the 3 assigned study days. For lunch, participants consumed 1 of 3 test meals: Control (C), avocado-free; Avocado Inclusive (AI); or, Avocado Added (AA) (see below). The standardized breakfast and the lunch test meals were designed to deliver the recommended levels of macronutrients according to the Acceptable Macronutrient Distribution Ranges developed by the Food and Nutrition Board of the Institute of Medicine [14]. The participants consumed 13-14\% energy from protein, 49-51\% energy from carbohydrate, and 35-38\% energy from fat at the three lunch test meals. Further, the $\mathrm{C}$ and AI lunch meals delivered 35\% of the participant's daily energy needs and the AA lunch meal provided $41 \%$ of the daily energy needs (Table 1). All foods were precisely measured or weighed to the nearest gram using a digital scale and the meals were matched for taste and appearance. Participants were permitted to drink water with and between meals on the three study days according to their typical pattern of water intake.

The standardized breakfast meal included orange juice, cornflakes, milk and a commercially prepared scone. The $\mathrm{C}$ test meal included a salad (mixed greens, cherry tomatoes, reduced fat Swiss cheese, Italian salad dressing), a refined grain French baguette and commercial chocolate chip cookies. Fresh, ripe Hass avocados (provided by the Hass Avocado Board) were sliced and included or added to the $\mathrm{C}$ test meal to produce the $\mathrm{AI}$ and AA test meals, respectively. The amount of avocado varied (range of $\sim 50$ to $90 \mathrm{~g})$ with the energy needs of the participant [75 $\mathrm{g}(\sim 1 / 2$ of an avocado) for the $2000 \mathrm{kcal}$ meal plan]. The portion sizes of the salad dressing and cookies were reduced in the AI test meal to match the energy and macronutrient content of the $\mathrm{C}$ test meal (Table 1 ).

The dinner buffet meal on the 3 study days was served 5 hours from the start of the lunch test meal and contained a variety of foods with pre-identified portion weight, macronutrient and calorie content. Participants were allowed to consume sweet and savory food options that differed in energy density in an ad libitum manner to allow for the assessment of postprandial food intake

Table 1 Percent of daily energy and macronutrient content of the 3 lunch test meals

\begin{tabular}{lccc}
\hline & Control & Avocado Inclusive & Avocado Added \\
\hline Energy, \% & 35 & 35 & 41 \\
Carbohydrate, \% & 51 & 50 & 49 \\
Protein, \% & 14 & 14 & 13 \\
Fat, \% & 35 & 36 & 38 \\
\hline
\end{tabular}

and dietary compensation. The number of portions of food items selected and consumed by the participants was directly observed and written in a discreet manner by a senior investigator and trained research staff. To supplement the written documentation of the food items taken and leftovers remaining on the plate, a hidden video camera was utilized to record the foodservice delivery process. The leftovers were photographed using a digital camera and weighed to the nearest gram using a digital scale. Two research assistants separately compared the written documentation with the videotape recording, still photos and weight of leftovers to produce a record of food intake for each participant. If any discrepancy existed between the two researcher's records, a senior investigator reviewed all of the data sources to determine the most valid measurement of dietary intake.

Pre-portioned evening snacks were provided to participants at the conclusion of the ad libitum dinner meal and participants were asked to record any snacks consumed after leaving the research kitchen until going to bed or until midnight. Participants were contacted by phone the following morning by study personnel for a self-report of the intake of pre-portioned evening snacks. The energy and macronutrient intake subsequent to the lunch test meals was assessed based on the observed food consumed at the ad libitum dinner meal and from the participant's selfreported consumption of pre-portioned evening snacks.

\section{Visual analog scales}

By means of a mark on $100 \mathrm{~mm}$ line visual analog scales (VAS), participants rated their appetite sensations (hunger, fullness, satisfied, desire for a meal, and prospective food consumption). The VAS was completed before lunch and at approximately 30, 60, 90, 120, 180 and 300 minutes following the lunch test meal on each study day. The five scales were anchored at the low end with the most negative feelings (e.g. not at all) and opposing terms at the high end (e.g. extremely high).

\section{Sample collection and laboratory assessment}

On the 3 assigned study days, participants arrived in the morning to the Nutrition Research Laboratory (NRL) after a 12-hour overnight fast for a baseline blood draw to measure glucose and insulin concentrations. Participants were free to engage in their normal morning routines but were instructed to return to the NRL by noon. The lunch test meal (C, AI or AA) was ingested within a 30 minute time period and additional blood samples were taken at approximately 30, 60, 90, 120 and 180 minutes following the start of the lunch test meal.

Venous blood samples were drawn and collected into vacutainer tubes (Becton Dickinson, Franklin Lakes, NJ) and centrifuged at $1500 \mathrm{x} \mathrm{g}$ at $4^{\circ} \mathrm{C}$ for $10 \mathrm{~min}$. Serum and plasma were separated, aliquoted and frozen at $-80^{\circ} \mathrm{C}$ until 
analyzed. Serum glucose was assayed with the glucoseoxidase-peroxidase enzymatic assay using kits supplied by Cayman Chemical (Ann Arbor, MI). Serum insulin was assayed using ELISA kits supplied by ALPCO Diagnostics (Salem, NH).

\section{Statistical methods}

Sample size, power calculations and statistical analysis were performed utilizing SAS version 9.3 (SAS Institute, Cary, NC). All tests were two-sided and a value of $P<$ 0.05 was considered significant. Under good experimental laboratory conditions, a sample size of 20 to 25 participants has been shown to be adequate to denote a $10 \%$ difference in AUC appetite ratings, which is considered to be a reasonable difference [15]. The target accrual was 28 participants to allow for a $10 \%$ dropout rate, which has been the observed dropout rate for our prior short-term feeding studies. A mixed model statistical approach was used to compare differences among the 3 test meals adjusting for study periods as fixed effects and treating participants as random effects. When significant findings were observed, Tukey post-hoc testing was performed to further elucidate the differences between the 3 test meals.

The weighted mean dietary intake of avocado was computed based on the number of participants assigned to each of the three aforementioned energy levels. The dietary compensation score across the $\mathrm{C}$ and AA test meals was calculated at the individual level using the following equation [6]: Percent Dietary Compensation $=($ Intake without load, C)-(Intake with load, AA)/Energy content of load $x$ 100. More specifically, the dietary compensation at dinner was computed at the individual level by subtracting a subject's dinner intake after the $\mathrm{C}$ lunch test meal minus the same subject's dinner intake on the day of the AA lunch test meal, divided by the energy (or macronutrient) from the avocado consumed.

Two trained research assistants measured the VAS data to the nearest $0.1 \mathrm{~cm}$ and any discrepancy was resolved by a senior investigator. To compute the area under the curve (AUC) from zero to $x$ hours $\left(\mathrm{AUC}_{(0-\mathrm{xh})}\right)$, the minimum value of each subjectively reported VAS scale (in $\mathrm{mm}$ ) over time was determined at the individual level and then the AUC above the minimum value was calculated using the linear trapezoidal rule. The AUC is reported as $\mathrm{mm} \mathrm{x}$ minutes and was constructed by plotting the subjective values between 0 to $100 \mathrm{~mm}$ over time (minute) for each of the five VAS questions.

The VAS was completed and blood samples were scheduled to be taken at approximately 30,60, 90, 120, and 180 minutes following the test meal. The exact time for each individual VAS and blood sample collection was recorded and these times were used for the analysis. Curved lines were generated to show the area under the curve for glucose and insulin (Figure 1) and the five VAS questions (Figure 2), which better represent the reality of the study data collection and analysis.

Data are expressed as adjusted mean \pm SE unless otherwise noted.

\section{Results}

The 26 participants that completed the study consisted of 16 women and 10 men with a mean \pm SD age $40.8 \pm$ 11.0 years and BMI $28.1 \pm 2.4 \mathrm{~kg} / \mathrm{m}^{2}$. Ten participants consumed the $1600 \mathrm{kcal}$ meal plan, nine consumed the

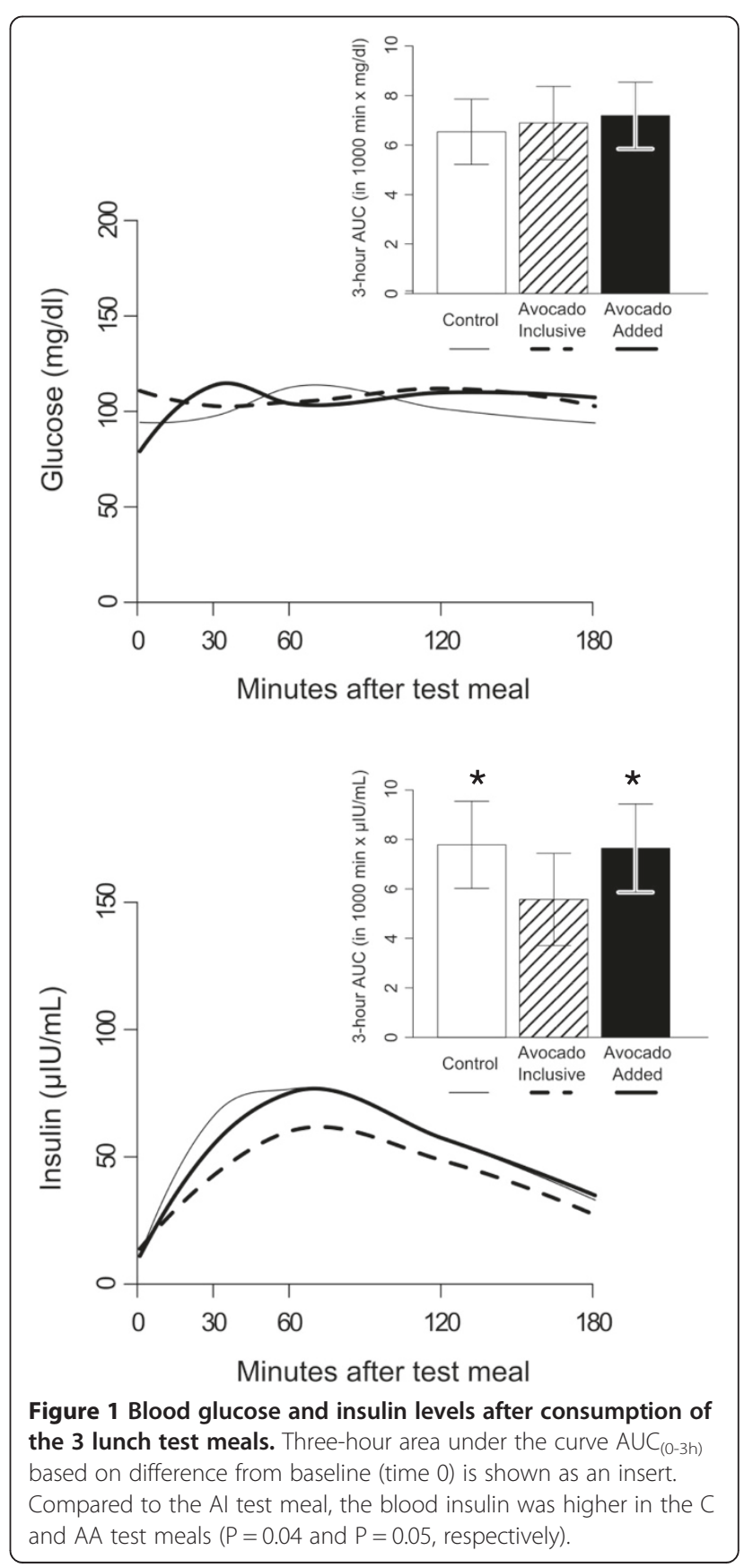




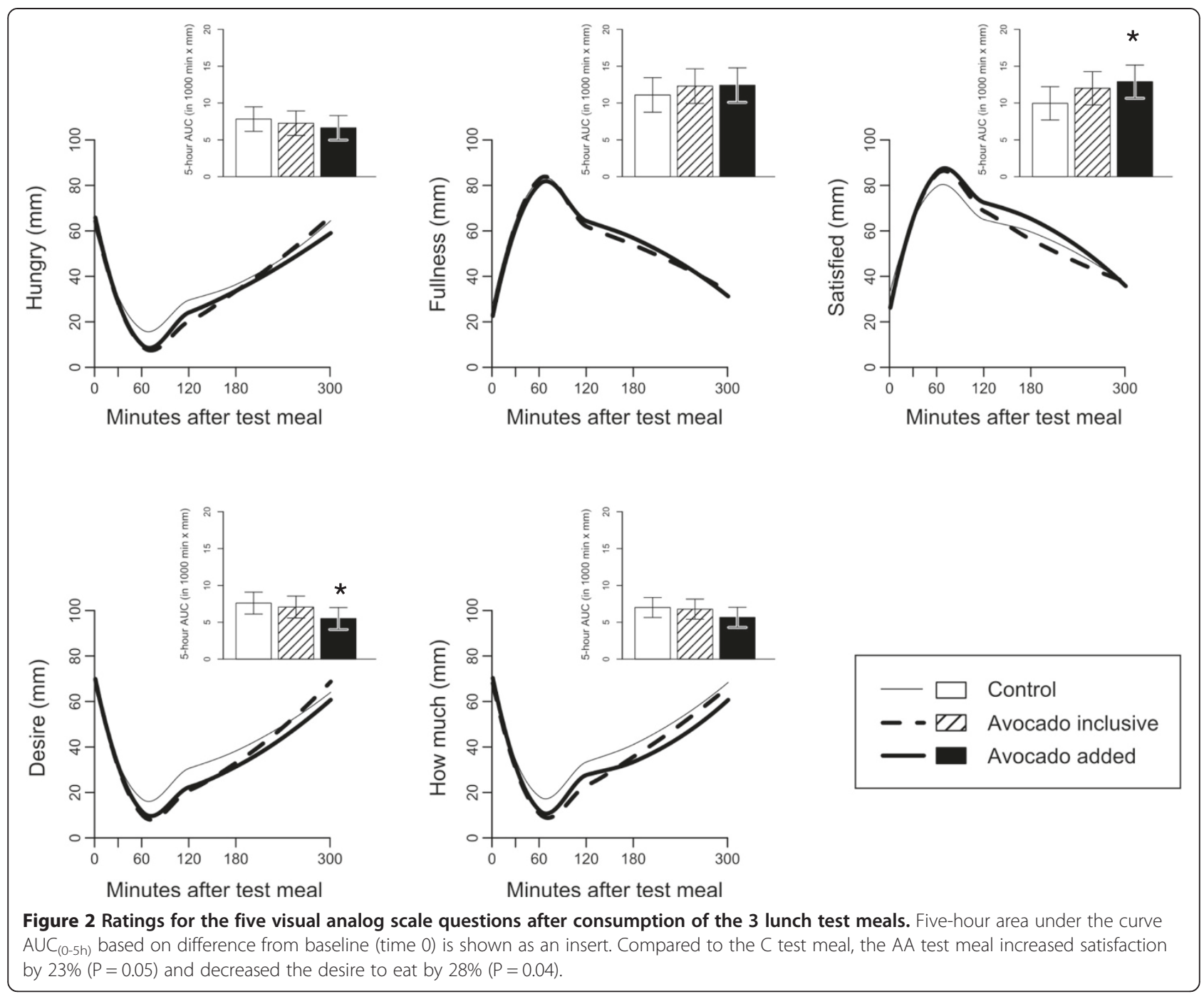

$2000 \mathrm{kcal}$ meal plan and seven consumed the $2400 \mathrm{kcal}$ meal plan. The weighted mean dietary intake of avocado was 67.5 grams, which contained $112 \mathrm{kcal}, 1.3 \mathrm{~g}$ protein, $5.6 \mathrm{~g}$ carbohydrate and $10.4 \mathrm{~g}$ fat.

\section{Blood glucose and insulin changes}

There were no significant differences between the 3 lunch test meals for $\mathrm{AUC}_{(0-3 \mathrm{~h})}$ blood glucose (Figure 1). Compared to the $\mathrm{AI}$ test meal, the $\mathrm{AUC}_{(0-3 \mathrm{~h})}$ for blood insulin was higher in the $\mathrm{C}$ and AA test meals $(\mathrm{P}=0.04$ and $\mathrm{P}=0.05$, respectively). Difference in blood insulin levels between treatments were observed at the $30 \mathrm{mi}-$ nute time point $(\mathrm{P}=0.04)$ as follows: adjusted mean $(95 \% \mathrm{CI})$ for $\mathrm{C}=54 \mu \mathrm{IU} / \mathrm{ml}(39,74)$; $\mathrm{AI}=34 \mu \mathrm{IU} / \mathrm{ml}(25$, 47); and, AA $42 \mu \mathrm{IU} / \mathrm{ml}(30,57)$.

\section{Visual analog scale changes}

There were significant differences in the $\mathrm{AUC}_{(0-5 \mathrm{~h})}$ for the self-reported subjective feelings of satisfaction $(P=0.04)$ and desire to eat $(\mathrm{P}=0.05)$ in the mixed model analysis (Figure 2). Post-hoc testing revealed that compared to the C test meal, the AA test meal increased satisfaction by $23 \%$ $(\mathrm{P}=0.05)$ and decreased the desire to eat by $28 \%(\mathrm{P}=0.04)$ for the $\mathrm{AUC}_{(0-5 \mathrm{~h})}$. For the $\mathrm{AUC}_{(0-3 \mathrm{~h})}$, the AA test meal increased satisfaction by $26 \%(P=0.02)$ and decreased the desire to eat by $40 \%(\mathrm{P}=0.01)$ as compared to the $\mathrm{C}$ test meal (Table 2). Additionally, the AI test meal showed a tendency towards increasing satisfaction by $22 \% \mathrm{P}=0.07$ ) as compared to the $\mathrm{C}$ test meal for the $\mathrm{AUC}_{(0-3 \mathrm{~h})}$. Lastly, the five measurements of appetite sensation tended to converge 5 hours after the lunch test meal.

Intake at the ad libitum dinner meal and evening snack Dietary intake at the subsequent ad libitum dinner meal and evening snack after each of the 3 lunch test meals was equivalent for total energy, macronutrients and percent energy from the macronutrients (Table 3). Compared to the $\mathrm{C}$ test meal, the percent dietary compensation for the 
Table 2 Three-hour area under the curve $A C_{(0-3 h)}^{a}$ based on difference from baseline (time 0 ) for the five visual analog scale questions between the 3 lunch test meals

\begin{tabular}{|c|c|c|c|c|c|c|c|c|c|}
\hline \multirow[b]{2}{*}{ Question } & \multicolumn{2}{|c|}{ Control } & \multicolumn{3}{|c|}{ Avocado Inclusive } & \multicolumn{3}{|c|}{ Avocado Added } & \multirow[b]{2}{*}{ P-value } \\
\hline & Mean $^{\mathbf{b}}$ & $\overline{\mathrm{SE}^{\mathrm{c}}}$ & Mean $^{\mathbf{b}}$ & Difference $^{d}$ & P-value ${ }^{\mathrm{e}}$ & Mean $^{\mathbf{b}}$ & Difference $^{d}$ & P-value ${ }^{\mathrm{e}}$ & \\
\hline How hungry are you? & 3105 & 394 & 2358 & $-24 \%$ & 0.30 & 2418 & $-22 \%$ & 0.36 & 0.26 \\
\hline How strong is your feeling of fullness? & 7249 & 771 & 8107 & $+11 \%$ & 0.64 & 8189 & $+11 \%$ & 0.58 & 0.55 \\
\hline How satisfied are you? & 6340 & 726 & 8149 & $+22 \%$ & 0.07 & 8562 & $+26 \%$ & 0.02 & 0.02 \\
\hline How strong is your desire to eat? & 2993 & 319 & 2263 & $-24 \%$ & 0.16 & 1798 & $-40 \%$ & 0.01 & 0.01 \\
\hline How much do you think you can eat? & 2641 & 318 & 2185 & $-17 \%$ & 0.45 & 2031 & $-23 \%$ & 0.24 & 0.25 \\
\hline
\end{tabular}

${ }^{\mathrm{a}}$ The AUC is reported as $\mathrm{mm} \times$ minutes and was constructed by plotting the subjective values between 0 to $100 \mathrm{~mm}$ over time (minute) for each of the five VAS questions.

${ }^{\mathrm{b}}$ Adjusted mean from the mixed model analysis.

${ }^{c}$ Common standard error (SE) for all of the adjusted means.

${ }^{\mathrm{d}}$ Percent difference compared to Control lunch test meal.

ep-value compared to Control lunch test meal.

${ }^{\mathrm{f}} \mathrm{P}$-value for diet effect from the mixed model analysis.

AA test meal for energy, protein, carbohydrate and fat was $66 \%, 235 \%, 118 \%$ and $36 \%$, respectively.

\section{Discussion}

The results of this study suggest that the addition of $\sim 1 / 2$ of a Hass avocado at a lunch meal can influence post-ingestive satiety over a subsequent 3 hour and 5 hour period in overweight and moderately obese adults. Specifically, adding avocado to a lunch meal yielded a $23 \%$ increase in satisfaction $(\mathrm{P}=0.05)$ and a $28 \%$ decreased desire to eat $(\mathrm{P}=0.04)$ over a subsequent 5 hour period as compared to the avocadofree control lunch meal. Also, adding avocado to a lunch meal yielded a $26 \%$ increase in satisfaction $(\mathrm{P}=0.02)$ and $40 \%$ decreased desire to eat $(\mathrm{P}=0.01)$ as compared to the avocado-free control lunch meal over a 3 hour period. However, an additional $112 \mathrm{kcal}$ was contained in the avocado, which may have accounted for the observed increased satisfaction and decreased desire to eat. Further, a $24 \%$ decreased desire to eat $(\mathrm{P}=0.16)$ and $22 \%$ increase in satisfaction $(\mathrm{P}=0.07)$ was observed over a 3 hour period after consumption of the isocaloric avocado inclusive lunch test meal as compared to the avocado-free control lunch meal. However, the changes in all five measurements of appetite sensation tended to taper off after 5 hours.

Energy intake at the subsequent ad libitum dinner meal and evening snack and dietary compensation did not differ between the 3 lunch test meals, which may have been due to the 5 hour time interval between the lunch test meal and ad libitum dinner meal. De Graaf and Hulshof [16] have previously reported that the weight or amount of food in a preload affects subsequent appetite and food intake for only up to two hours after the preload. These findings are consistent with the findings of equivalent energy intake at the subsequent dinner meal and evening snack in the current study, yet inconsistent with changes in two specific measures of appetite sensation that we observed at both 3 and 5 hours for the avocado added test meal. Further, Flint et al. [15] has reported that an $8-10 \%$ difference in the response magnitude relative to control in food intake or satiety score (AUC) is of practical relevance. We found differences of practical relevance for all five appetite

Table 3 Intake from the dinner meal and evening snack after the $\mathbf{3}$ lunch test meals

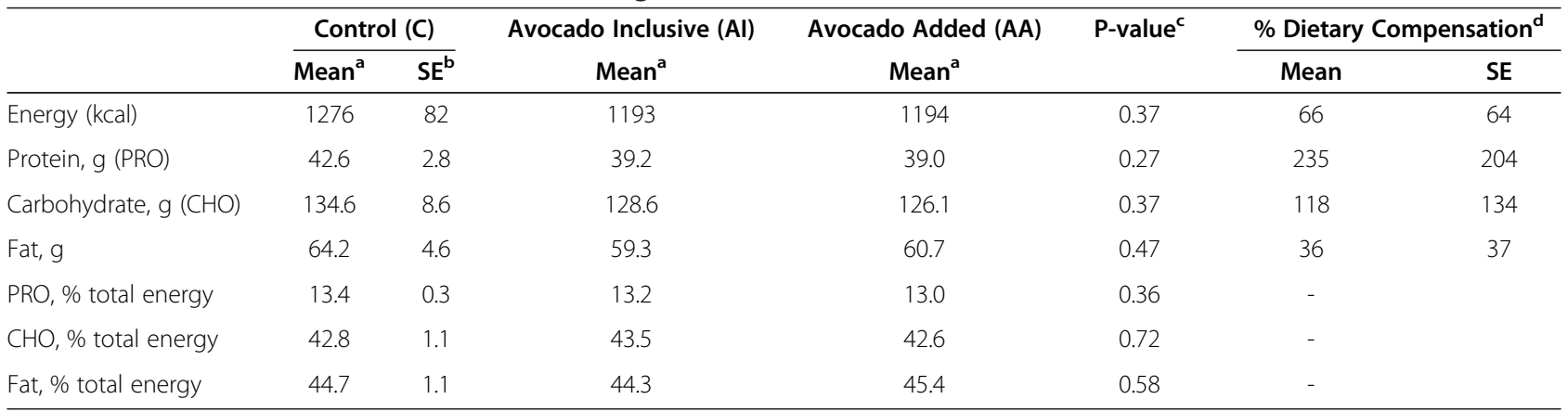

${ }^{a}$ Adjusted mean based on the mixed model analysis.

${ }^{\mathrm{b} C o m m o n}$ standard error (SE) for all of the adjusted means.

${ }^{\mathrm{C}} \mathrm{P}$-value for diet effect from the mixed model analysis.

P-value for diet effect from the mixed model analysis.
d\%Dietary Compensation=(Intake without load,, ) (Intake with load, $A A) \times 100$.

[Note: The \% dietary compensation at dinner was computed at the individual level by subtracting a subject's dinner intake after the $\mathrm{C}$ lunch test meal minus the same subject's dinner intake on the day of the AA lunch test meal, divided by the energy (or macronutrient) from the avocado consumed]. 
sensation measurements between the $C$ versus the AI and AA interventions ranging between $11-24 \%$ and 11 $40 \%$, respectively. However, we did not find a statistically significant difference for hunger, fullness or prospective food consumption between the 3 test meals.

Our overweight participants partially compensated for energy (66\%) and fat (36\%) intake and overcompensated for protein (235\%) and carbohydrate $(118 \%)$ at a subsequent ad libitum dinner meal and evening snack when avocado (weighted mean energy $=112 \mathrm{kcal}$ ) was added to the lunch meal. Thus, the majority of the energy provided by the addition of avocado to the diet was offset by dietary adjustments at the ad libitum dinner meal and evening snack. Others have reported that individual daily energy intake can vary by 20 to 30 percent, and that short-term dietary manipulations of less than $\sim 400 \mathrm{kcal}$ may not heavily influence dietary energy compensation $[17,18]$, which may have been one of the reasons for the equivalent subsequent energy intake at the dinner meal and evening snack between the 3 study days.

There are two potential ways a whole food can be incorporated into a meal, addition or isocaloric replacement. Addition is when the food is simply added to a meal, which results in an increase in nutrients and total energy, whereas isocaloric replacement occurs when the food is included and other foods are simultaneously decreased or eliminated to compensate for the overall energy content of the meal. It is worth noting that the $\mathrm{AUC}_{(0-3 \mathrm{~h})}$ for blood glucose in the current study was equivalent between the 3 lunch test meals despite the additional mean energy (112 kcal) content and additional $\sim 7$ g carbohydrate in the AA lunch test meal. Avocados contain a unique seven carbon sugar (D-manno-heptulose) that does not contribute energy, and some believe it may support blood glucose control and weight management by reducing glycolysis via hexokinase inhibition [19]. Additionally, 30 minutes after the start of the lunch test meal the inclusion and addition of avocado significantly attenuated the rise in blood insulin levels by $37 \%$ and $22 \%$, respectively $(P=0.04)$. Avocados are rich in antioxidants (e.g. polyphenolic compounds), which others have shown to be effective in improving insulin sensitivity in an overweight cohort [20]. Hence, including or adding avocado to a dietary pattern may assist in ameliorating the postprandial dysfunction in glucose homeostasis that may be present in overweight individuals.

The $\mathrm{AUC}_{(0-3 \mathrm{~h})}$ for blood insulin was lower in the AI test meal compared to both the $\mathrm{C}$ and the AA test meals, however this biological parameter did not significantly influence the five appetite sensation measurements between the $\mathrm{AI}$ and avocado-free $\mathrm{C}$ test meal $(\mathrm{P}=0.07$ to $\mathrm{P}=0.64$ ). It is worth noting that the five appetite sensation measurements for both the AI and AA test meals went in a favorable and similar direction, and borderline significant findings were found between the $\mathrm{AI}$ and $\mathrm{C}$ test meal in the context of increased satisfaction $(P=0.07)$ and a tendency existed towards reducing the desire to eat $(\mathrm{P}=$ $0.16)$.

Insulin and the incretin hormones covary in response to elevated postprandial glucose levels [21], which makes it challenging to uphold the glucostatic theory proposed by Mayer [22]. Andersen et al. have observed that postprandial levels of blood glucose are inversely associated with self-reported appetite and food intake [23], however others have shown no association between satiety and blood glucose levels using an intravenous carbohydrate infusion [24]. Thus, it is plausible that the incretin hormones were influenced by the fat and fiber from the addition of avocado to the AA test meal, which yielded an increase in satisfaction and a reduction in the desire to eat. Although fat delays gastric emptying, some studies have shown that protein in the diet has the most potent action on satiety followed by carbohydrate, and fat the least $[25,26]$. However, it is important to note that studies designed to evaluate the satiety level of fat usually add fat to a meal in the form of oil or shortening, which increases the energy density of the meal without appreciably altering the volume of the meal. Thus, the low satiating effect of fat found in some studies may have been mediated exclusively by the increase in energy density.

It is also worth noting that the intake at the ad libitum dinner and evening snack was similar between the AI and AA lunch test meals, and that the inclusion of avocado at a meal along with a concurrent reduction in other foods containing similar macronutrients favorably reduced the subsequent energy intake by $83 \mathrm{kcal}(6.5 \%)$, and reduced the protein, carbohydrate and fat intake by $3.4 \mathrm{~g}, 6.0 \mathrm{~g}$ and $4.9 \mathrm{~g}$, respectively. Avocados are a rich source of monounsaturated fatty acids, which are preferentially oxidized and increase thermogenesis as compared to polyunsaturated and saturated fatty acids. Thus, the inclusion of avocados to a dietary meal pattern may have additional implications in weight management in an overweight population.

Although we found a significant reduction in insulin levels and favorable changes in two specific measures of appetite sensation for the AI and AA lunch test meals, respectively, we did not observe any behavioral change in dietary intake at the subsequent ad libitum dinner meal and evening snack between the 3 test meals. However, this latter null finding should not be over-interpreted as the data presented in this study are for 3 separate days (one week apart) and additional dietary energy compensation is plausible over several days and weeks [27].

This study had several strengths and limitations. Our controlled "laboratory" type setting had high internal validity due to the high degree of sensitivity and control over the dietary intervention and study outcome measures. An 
additional strength is that we analyzed the AUC appetite sensation data as opposed to a single time point because analysis of individual time points is not physiologically independent and is prone to type 1 errors. A study limitation is that we did not measure dietary intake in-between the 3 assigned study days. An additional study limitation is that we provided a wide variety of foods at our ad libitum dinner buffet meal, which is at variance with the typical eating pattern of most individuals and is likely to delay satiation and facilitate increased food intake [28]. Lastly, we may have placed the participants in an atypical environment by not providing food to them for 5 hours.

\section{Conclusions}

This study showed that the addition of $\sim 1 / 2$ of a fresh Hass avocado to a lunch meal favorably increased satisfaction and reduced the desire to eat over a subsequent 3 hour and 5 hour period in an overweight and moderately obese adult population. When avocados were either added or included to a lunch meal, similarities in five measures of appetite sensation were found over a subsequent 3 hour period. Given that the peak satisfaction effect was found to be within 3 hours of the lunch test meal, subsequent studies should address the offering of snacks as this may be of importance to overweight and moderately obese adults that typically consume large snacks between meals. Therefore, the addition of $\sim 1 / 2$ of an avocado at a specific meal(s) may be a simple dietary intervention to consider for individuals that consume large snacks (e.g. excessive energy) between meals.

Both the inclusion and addition of avocado to a lunch meal attenuated the rise in postprandial blood insulin levels 30 minutes after the start of the lunch meal as compared to the avocado-free control lunch meal. Additionally, the inclusion of avocado to a lunch meal yielded a significant reduction in blood insulin levels over a 3 hour postprandial period. The attenuation in the rise of insulin in the avocado inclusive intervention is worthy of future exploration in persons with insulin resistance and type 2 diabetes mellitus to determine if avocado intake can favorably influence measures of glucose homeostasis. Lastly, a longer trial would be beneficial to evaluate the effects of daily avocado intake on measures of appetite sensation and weight management in free-living normal weight, overweight and obese adults.

\section{Competing interests}

The authors have no conflicts of interest or competing interests.

\section{Authors' contributions}

EH and JS designed the study. EH, MW and JS coordinated the study. MW and $\mathrm{EH}$ were responsible for data collection, analysis and quality control. $\mathrm{MW}, \mathrm{EH}, \mathrm{JS}$ and $\mathrm{KO}$ were involved in the statistical analyses. All authors contributed to the interpretation of data. MW wrote the first draft of the manuscript and all authors critically reviewed and revised the manuscript. JS obtained the funding for the study. All authors read and approved the final manuscript.

\section{Acknowledgements}

The funding for this study was supported by a grant from the Hass Avocado Board, which had no role in the design and conduct of the study; in the collection, analysis, and interpretation of the data; or in the preparation, review or approval of the manuscript.

\section{Author details}

'Department of Nutrition, School of Public Health, Loma Linda University, 24951 N. Circle Dr., Nichol Hall 1102, Loma Linda, CA 92350, USA.

${ }^{2}$ Department of Epidemiology and Biostatistics, Loma Linda University, Loma Linda, CA, USA.

Received: 17 August 2013 Accepted: 22 November 2013

Published: 27 November 2013

\section{References}

1. Giskes $K$, van Lenthe F, Avendano-Pabon M, Brug J: A systematic review of environmental factors and obesogenic dietary intakes among adults: are we getting closer to understanding obesogenic environments? Obes Rev 2011, 12:e95-e106.

2. Ouyang H, Yin J, Chen JD: Gastric or intestinal electrical stimulation-induced increase in gastric volume is correlated with reduced food intake. Scand J Gastroenterol 2006, 41:1261-1266.

3. Rolls BJ: The relationship between dietary energy density and energy intake. Physiol Behav 2009, 97:609-615.

4. Jones PJ, Schoeller DA: Polyunsaturated:saturated ratio of diet fat influences energy substrate utilization in the human. Metabolism 1988, 37:145-151.

5. Piers LS, Walker KZ, Stoney RM, Soares MJ, O'Dea K: The influence of the type of dietary fat on postprandial fat oxidation rates: monounsaturated (olive oil) vs saturated fat (cream). Int J Obes Relat Metab Disord 2002, 26:814-821.

6. DiMeglio DP, Mattes RD: Liquid versus solid carbohydrate: effects on food intake and body weight. Int J Obes Relat Metab Disord 2000, 24:794-800.

7. Dreher ML, Davenport AJ: Hass avocado composition and potential health effects. Crit Rev Food Sci Nutr 2013, 53:738-750.

8. Bes-Rastrollo M, van Dam RM, Martinez-Gonzalez MA, Li TY, Sampson LL, Hu FB: Prospective study of dietary energy density and weight gain in women. Am J Clin Nutr 2008, 88:769-777.

9. Fulgoni VL 3rd, Dreher M, Davenport AJ: Avocado consumption is associated with better diet quality and nutrient intake, and lower metabolic syndrome risk in US adults: results from the National Health and Nutrition Examination Survey (NHANES) 2001-2008. Nutr J 2013, 12:1.

10. Burton-Freeman B: Dietary fiber and energy regulation. J Nutr 2000, 130:272S-275S.

11. Juvonen KR, Purhonen AK, Salmenkallio-Marttila M, Lahteenmaki L, Laaksonen DE, Herzig KH, Uusitupa MI, Poutanen KS, Karhunen LJ: Viscosity of oat bran-enriched beverages influences gastrointestinal hormonal responses in healthy humans. J Nutr 2009, 139:461-466.

12. Flint A, Gregersen NT, Gluud LL, Moller BK, Raben A, Tetens I, Verdich C, Astrup A: Associations between postprandial insulin and blood glucose responses, appetite sensations and energy intake in normal weight and overweight individuals: a meta-analysis of test meal studies. $\mathrm{Br} J$ Nutr 2007, 98:17-25.

13. Popkin BM, Duffey KJ: Does hunger and satiety drive eating anymore? Increasing eating occasions and decreasing time between eating occasions in the United States. Am J Clin Nutr 2010, 91:1342-1347.

14. Food and Nutrition Board, Institute of Medicine, National Academies: Dietary Reference Intakes for Energy, Carbohydrate, Fiber, Fat, Fatty Acids, Cholesterol, Protein, and Amino Acids (2002/2005). Dietary Reference Intakes (DR/s): Acceptable Macronutrient Distribution Ranges. Available at: http://www.nap.edu/openbook. php?record_id=10490\&page=1325. Accessed July 10, 2012.

15. Flint A, Raben A, Blundell JE, Astrup A: Reproducibility, power and validity of visual analogue scales in assessment of appetite sensations in single test meal studies. Int J Obes Relat Metab Disord 2000, 24:38-48.

16. de Graaf C, Hulshof T: Effects of weight and energy content of preloads on subsequent appetite and food intake. Appetite 1996, 26:139-151.

17. Beaton GH, Milner J, Corey P, McGuire V, Cousins M, Stewart E, de Ramos M, Hewitt D, Grambsch PV, Kassim N, Little JA: Sources of variance in 24-hour dietary recall data: implications for nutrition study design and interpretation. Am J Clin Nutr 1979, 32:2546-2559. 
18. Sukhatme PV, Margen S: Autoregulatory homestatic nature of energy balance. Am J Clin Nutr 1982, 35:355-365.

19. Roth G, Hayek MSM, Davenport G, Arking R, Bartke A, Bonkowki M, Ingram D: Mannoheptulose glycolytic inhibitor and novel calorie restriction mimetic. In Experimental Biology. New Orleans, LA: Abstract \# 553.1; 2009

20. Skulas-Ray AC, Kris-Etherton PM, Teeter DL, Chen CY, Vanden Heuvel JP, West SG: A high antioxidant spice blend attenuates postprandial insulin and triglyceride responses and increases some plasma measures of antioxidant activity in healthy, overweight men. J Nutr 2011, 141:1451-1457.

21. Holt SH, Brand Miller JC, Petocz P: Interrelationships among postprandial satiety, glucose and insulin responses and changes in subsequent food intake. Eur J Clin Nutr 1996, 50:788-797.

22. Mayer J: Regulation of energy intake and the body weight: the glucostatic theory and the lipostatic hypothesis. Ann N Y Acad Sci 1955, 63:15-43.

23. Anderson GH, Catherine NL, Woodend DM, Wolever TM: Inverse association between the effect of carbohydrates on blood glucose and subsequent short-term food intake in young men. Am J Clin Nutr 2002, 76:1023-1030

24. Lavin JH, Wittert G, Sun WM, Horowitz M, Morley JE, Read NW: Appetite regulation by carbohydrate: role of blood glucose and gastrointestinal hormones. Am J Physiol 1996, 271:E209-E214.

25. Blundell JE, MacDiarmid Jl: Fat as a risk factor for overconsumption: satiation, satiety, and patterns of eating. J Am Diet Assoc 1997, 97:S63-S69.

26. Rolls BJ, Kim-Harris S, Fischman MW, Foltin RW, Moran TH, Stoner SA: Satiety after preloads with different amounts of fat and carbohydrate: implications for obesity. Am J Clin Nutr 1994, 60:476-487.

27. Tarasuk V, Beaton $\mathrm{GH}$ : The nature and individuality of within-subject variation in energy intake. Am J Clin Nutr 1991, 54:464-470.

28. Hetherington MM, Foster R, Newman T, Anderson AS, Norton G. Understanding variety: tasting different foods delays satiation. Physiol Behav 2006, 87:263-271.

doi:10.1186/1475-2891-12-155

Cite this article as: Wien et al:: A randomized $3 \times 3$ crossover study to evaluate the effect of Hass avocado intake on post-ingestive satiety, glucose and insulin levels, and subsequent energy intake in overweight adults. Nutrition Journal 2013 12:155.

\section{Submit your next manuscript to BioMed Central and take full advantage of:}

- Convenient online submission

- Thorough peer review

- No space constraints or color figure charges

- Immediate publication on acceptance

- Inclusion in PubMed, CAS, Scopus and Google Scholar

- Research which is freely available for redistribution 